\title{
Interações medicamentosas associadas a Ginkgo biloba L.: Revisão de literatura
}

\author{
Drug interactions associated to Ginkgo biloba L.: Literature review \\ Interacciones medicamentosas asociadas con Ginkgo biloba L.: revisión de la literatura
}

Recebido: 27/04/2021 | Revisado: 50/05/2021 | Aceito: 09/05/2021 | Publicado: 23/05/2021

\author{
Ana Caroline da Silva \\ ORCID: https://orcid.org/0000-0002-4801-6322 \\ AESPI-Associação de Ensino Superior do Piauí, Brasil \\ E-mail: annakaroline.199848@gmail.com \\ Joseana Martins Soares de Rodrigues Leitão \\ ORCID: https://orcid.org/0000-0003-1794-4916 \\ AESPI-Associação de Ensino Superior do Piauí, Brasil \\ E-mail: joseanaleitao@hotmail.com
}

\begin{abstract}
Resumo
No último século o progresso dos estudos relacionados a medicamentos proporcionaram o combate a alguns males que atingem a humanidade, entretanto estima-se que $80 \%$ da população busca terapias alternativas, pois a dificuldade em ter acesso a serviços básicos de saúde, seja pela alta demanda de pacientes ou até mesmo ao alto custo para aquisição de medicamentos faz com que busquem com mais frequência tratamentos com fitoterápicos que estão entre os recursos da medicina complementar e vem sendo utilizado há muito tempo pela população O Ginkgo é utilizado há séculos pela medicina tradicional chinesa para a melhoria do estado de alerta. A pesquisa buscou realizar um levantamento bibliográfico sobre as principais interações entre fármacos e medicamentos fitoterápicos elaborados à base de ginkgo (Ginkgo biloba L.). O estudo foi executado através de uma revisão sistemática, considerando a relevância do tema, buscando conhecer sob o olhar de alguns autores bem como analisar como e quais as interações medicamentosas do fitoterápico G. biloba. A elaboração da pesquisa teve por ferramenta embasadora material já publicado sobre o tema; artigos científicos em publicações periódicas e disponíveis nos seguintes bancos de dados: Scielo e Pubmed, sendo considerados os artigos publicados entre 2010-2020, que relatavam sobre as possíveis interações medicamentosas do fitoterápico G. biloba. Da pesquisa bibliográfica efetuada obteve um total 497 artigos publicados entre os anos de 2010 a 2020. Após leitura, selecionou-se sete trabalhos, iniciando leituras exaustivas dos textos, fazendo uma síntese de cada artigo e categorizando os tipos de interações medicamentosas relacionadas a $G$. biloba.
\end{abstract}

Palavras-chave: Revisão de literatura; Fitoterápicos; Fármacos.

\begin{abstract}
In the last century, the progress of studies related to medications has made it possible to combat some ills that affect humanity, however it is estimated that $80 \%$ of the population seeks alternative therapies, since the difficulty in having access to basic health services, either due to high the demand from patients or even the high cost of purchasing drugs makes them more often seek treatments with herbal medicines that are among the resources of complementary medicine and have been used for a long time by the population. Ginkgo has been used for centuries by traditional Chinese medicine to improve alertness. The research sought to carry out a bibliographic survey on the main interactions between drugs and herbal medicines made from ginkgo (Ginkgo biloba L.). The study was carried out through a systematic review, considering the relevance of the theme, seeking to know under the eyes of some authors as well as analyzing how and what are the drug interactions of the herbal medicine G. biloba. The elaboration of the research was based on material already published on the topic; scientific articles in periodic publications and available in the following databases: Scielo and Pubmed, considering articles published between 2010-2020, which reported on the possible drug interactions of the herbal medicine G. biloba. From the bibliographic research carried out, a total of 497 articles were published between the years 2010 to 2020. After reading, seven papers were selected, starting exhaustive readings of the texts, making a synthesis of each article and categorizing the types of drug interactions related to $G$. biloba.
\end{abstract}

Keywords: Literature review; Phytotherapics; Drugs.

\section{Resumen}

En el último siglo, el avance de los estudios relacionados con los medicamentos ha ayudado a combatir algunos males que afectan a la humanidad, sin embargo se estima que el $80 \%$ de la población busca terapias alternativas, ya que la dificultad para acceder a los servicios básicos de salud, ya sea por la alta demanda de los pacientes o incluso el alto costo de la compra de medicamentos hace que ellos busquen con mayor frecuencia tratamientos con medicamentos a base de hierbas que se encuentran entre los recursos de la medicina complementaria y han sido utilizados durante mucho tiempo por la población. El ginkgo ha sido utilizado durante siglos por los chinos tradicionales. medicina para 
mejorar el estado de alerta. La investigación buscó realizar un relevamiento bibliográfico sobre las principales interacciones entre fármacos y medicamentos herbarios elaborados a base de ginkgo (Ginkgo biloba L.). El estudio se llevó a cabo mediante una revisión sistemática, considerando la relevancia del tema, buscando conocer bajo la mirada de algunos autores así como analizar cómo y cuáles son las interacciones farmacológicas de la fitoterapia G. biloba. La elaboración de la investigación se basó en material ya publicado sobre el tema; artículos científicos en publicaciones periódicas y disponibles en las siguientes bases de datos: Scielo y Pubmed, considerando los artículos publicados entre 2010-2020, que informaron sobre las posibles interacciones farmacológicas de la fitoterapia $G$. biloba. De la investigación bibliográfica realizada, se publicaron un total de 497 artículos entre los años 2010 a 2020. Luego de la lectura, se seleccionaron siete artículos, iniciando lecturas exhaustivas de los textos, haciendo una síntesis de cada artículo y categorizando los tipos de interacciones medicamentosas relacionadas. a G. biloba.

Palabras clave: Revisión de literatura; Fitoterápicos; Drogas.

\section{Introdução}

No último século o progresso dos estudos relacionados a medicamentos proporcionaram o combate a alguns males que atingem a humanidade, entretanto estima-se que $80 \%$ da população busca terapias alternativas, pois a dificuldade em ter acesso a serviços básicos de saúde, seja pela alta demanda de pacientes ou até mesmo ao alto custo para aquisição de medicamentos faz com que busquem com mais frequência tratamentos com fitoterápicos que estão entre os recursos da medicina complementar e vem sendo utilizado há muito tempo pela população (Nascimento-Junior, et al., 2016). As primeiras descobertas foram feitas por estudos arqueológicos em ruínas do Irã. Na China, em 3.000 a.C., já existiam farmacopéias que compilavam as ervas e as suas indicações terapêuticas.

As plantas são fábricas químicas para a biossíntese de uma enorme variedade de metabólitos secundários e, de fato, são esses metabólitos que formam a base de muitos medicamentos farmacêuticos, bem como remédios de ervas derivados de plantas medicinais. Os diferentes constituintes químicos das plantas medicinais possuem atividades biológicas que podem melhorar a saúde (Hassan, 2012; Sanchita \& Sharma, 2018). A Organização Mundial de Saúde (OMS) divulgou que 60-85\% da população dos países em desenvolvimento dependiam das plantas medicinais como única forma de acesso aos cuidados da saúde (Barreto Junior, et al., 2005).

As plantas medicinais têm sido utilizadas para fins terapêuticos desde a antiguidade e continuam a ser utilizadas até hoje para prevenir, curar e tratar doenças (Wachtel - Galor \& Benzie, 2011). Mesmo com a evolução da indústria farmacêutica, a cultura do uso das plantas e o conhecimento sobre seus benefícios foram preservados, o que demonstra sua importância e eficácia (Niehues, 2011).

A árvore de Ginkgo é nativa do Japão, Coréia e China, distribuída em várias regiões da América, Europa, áreas temperadas da Índia, Nova Zelândia e Argentina (Belwal, et al., 2019). Foi introduzido para Europa em torno de 1.730 e é geralmente plantada como planta medicinal, especialmente na Alemanha e na França. O gênero Ginkgo contém apenas uma espécie, Ginkgo biloba L., geralmente chamada de "fóssil vivo". Não há variabilidade na morfologia, hábito do caule ou composição química; portanto, é classificada em uma divisão separada, a Ginkgophyta (Zhang, et al., 2016). O Ginkgo é utilizado há séculos pela medicina tradicional chinesa para a melhoria do estado de alerta (Viegas Júnior, et al., 2004). Suas folhas são usadas na prevenção da arteriosclerose, na formação de trombos, em cardiopatias isquêmicas e para diabetes mellitus (D’Ippolito, et al., 2005).

G. biloba é conhecida como uma planta valiosa para a humanidade há mais de 2000 anos e considerada um "fóssil vivo" (Singh, et al., 2008). Tem sido usada na medicina tradicional e estudos indicaram seus efeitos benéficos, incluindo antiinflamatórios, fotoprotetores, hepatoprotetores, cardioprotetores e antioxidantes (Priyanka, et al., 2017).

O uso de extratos padronizados da folha do Ginkgo, testados nos últimos 30 anos, comprovou os efeitos estudados, enquanto o uso das folhas frescas ou de extratos em baixas concentrações não apresentaram os efeitos desejados (TUROLLA; Nascimento, 2006). O extrato do Ginkgo padronizado é denominado de Egb 761, contém porcentagens específicas de 
glicosídeos de ginkgoflavonas (24\%), correspondendo à fração flavonóica e terpenóides (6\%). A fração flavonóide (constituída pela miricetina, quercetina e rutina) é apontada como a responsável pelos efeitos antioxidantes do extrato (Macarenco, et al., 2001).

No Brasil, há uma boa aceitação por parte dos médicos da prescrição do Ginkgo, pelo fato da planta apresentar toda a sua farmacologia pré-clínica e clínica já realizada no exterior, tornando esse fitoterápico aceito como medicamento com todas as exigências de segurança e eficácia (Barata, 2005). Os médicos brasileiros prescrevem o uso do Ginkgo em casos de: labirintopatias, cefaléia, perturbações da memória e claudicação intermitente (Forlenza, 2003).

A fitoterapia pode ser descrita como o recurso de prevenção e tratamento de males através de plantas medicinais, sendo essa é a forma mais antiga e fundamental da medicina na terra. A palavra fitoterapia é derivada da palavra grega, Apeas phitos, que significa plantas e terapia que quer dizer tratamento. No objetivo de encontrar defesas naturais para o organismo a fitoterapia é notoriamente empregada em culturas africanas e indígenas, em monastérios no período medieval, sendo aplicada na forma de extratos in natura, manipulados e também hoje em dia industrializados (CRF-SP, 2013).

A utilização da natureza para fins terapêuticos é tão antiga quanto a civilização humana e, por muito tempo, produtos minerais, de plantas e animais foram fundamentais para a área da saúde. Historicamente, as plantas medicinais são importantes como fitoterápicos e na descoberta de novos fármacos, estando no reino vegetal a maior contribuição de medicamentos. (Rodriguez \& Amaral, 2012). O termo fitoterapia foi dado à terapêutica que utiliza os medicamentos cujos constituintes ativos são plantas ou derivados vegetais, e que tem a sua origem no conhecimento e no uso popular. As plantas utilizadas para esse fim são tradicionalmente denominadas medicinais (De Pasquale, 1984). A terapia com medicamentos de espécies vegetais é relatada em sistemas de medicinas milenares em todo o mundo, por exemplo, na medicina chinesa, tibetana ou indianaayurvédica.

A seleção de espécies vegetais para estudo farmacológico pode ser baseada no seu uso tradicional por sociedades tradicionais, no conteúdo químico e toxicidade, na seleção ao acaso ou pela combinação de vários critérios. Uma das estratégias mais comuns é o estudo da medicina tradicional e/ou popular em diferentes culturas, conhecida como etnofarmacologia. Estratégias de busca de medicamentos com base nessa linha de atuação têm sido aplicadas no tratamento de diferentes doenças, tais como o câncer (Kinghorn, 2003, Balunas \& Kinghorn, 2005).

A ideia errônea de que as plantas medicinais comercializadas são seguras já que são de fonte natural faz ser cada vez mais frequente o uso dessas. Além do mais, há a divulgação dessas informações pela mídia. Um fato preocupante é que as maiorias dos consumidores das plantas medicinais não informam ao médico, o que pode aumentar os riscos ao paciente, já que há várias interações já estudadas entre medicamentos e plantas medicinais, ou ainda ocorrer um erro de diagnóstico, causado por essas interações (Veiga, et al., 2005).

Cada vegetal pode ser tanto um alimento, ou ainda agir tanto como veneno ou como medicamento, que podem ser distintas na dose, via de administração ou a finalidade que será usada. Normalmente, os fitoterápicos são compostos por mais de uma espécie química, porém, esses deveriam ser submetidos às mesmas exigências de controle de qualidade que a dos medicamentos industrializados, fato que nem sempre é cumprido, já que é difícil estimar quais princípios ativos e quantidade estão presentes na amostra (França, et al., 2008).

Interações medicamentosas são tipos especiais de respostas farmacológicas, em que os efeitos de um ou mais medicamentos são alterados pela administração simultânea ou anterior de outros, ou através da administração concorrente com alimentos (Gahart \& Nazareno, 1997). As respostas decorrentes da interação podem acarretar potencialização do efeito terapêutico, redução da eficácia, aparecimento de reações adversas com distintos graus de gravidade ou ainda, não causar nenhuma modificação no efeito desejado do medicamento, Portanto, a interação entre medicamentos pode ser útil (benéfica), causar respostas desfavoráveis não previstas no regime terapêutico (adversa), ou apresentar pequeno significado clinico. (Oga 
\& Basile, 1994).

É frequentemente difícil detectar uma interação medicamentosa, sobretudo pela variabilidade observada entre pacientes. Não se sabe muito sobre os fatores de predisposição e de proteção que determinam se uma interação ocorre ou não, mas na prática ainda é muito difícil predizer o que acontecerá quando um paciente individual faz uso de dois fármacos que potencialmente interagem entre si.

Algumas interações medicamentosas apresentam potencial para causar danos permanentes, muitas são responsáveis por deterioração clínica do paciente - hospitalizações, aumento no tempo de internação, enquanto que outras são leves e não exigem medidas especiais. O desfecho de uma interação medicamentosa pode ser perigoso quando promove aumento da toxicidade de um fármaco. Por exemplo, pacientes que fazem uso de varfarina podem ter sangramentos se passarem a usar um anti-inflamatório não-esteróide (AINE) sem reduzir a dose do anticoagulante (OMS, 2006.).

O desfecho de uma interação medicamentosa pode ser perigoso quando promove aumento da toxicidade de um fármaco. Por exemplo, pacientes que fazem uso de varfarina podem ter sangramentos se passarem a usar um anti-inflamatório não-esteróide (AINE) sem reduzir a dose do anticoagulante.

Entre as plantas medicinais, G. biloba é considerada uma das mais famosas mundialmente, seu processo de produção é extremamente complexo e caro, tornando este extrato alvo de adulteração. Sua introdução da medicina ocorreu em 1965 pelo médico farmacêutico alemão Wilmar Schwabe através das suas pesquisas. Diversas atividades farmacológicas do extrato de suas folhas e/ou de seus componentes já foram documentadas, entre elas o efeito sobre a conduta, o aprendizado, a memória, a atividade cardiovascular, seu efeito sobre a circulação sanguínea e sua atividade antioxidante (Barnes, et al., 2005).

G. biloba parece ser a espécie viva de árvore mais antiga existente, e é uma das ervas medicinais mais vendidas na Europa e nos Estados Unidos, usada no tratamento de doenças cardiovasculares e do sistema nervoso central (thomas \& homas, 2004, Calixto, 2001). A folha de Ginkgo contém dois grupos de compostos dotados de propriedades farmacológicas interessantes: os flavonoides e os diterpenos. Os flavonoides são representados por vinte compostos, entre eles derivados heterosídeos de flavonóis e biflavonóis. Aos diterpenos está relacionada a capacidade em inibir o fator de agregação plaquetária (PAF) e aos flavonóis é atribuída uma atividade captadora de radicais livres (Schenkel, et al., 2000).

$\mathrm{O}$ extrato das folhas de Ginkgo é indicado para transtornos cognitivos, incluindo demência e insuficiência vascular cerebral, perda de memória recente, cefaléia, vertigens e zumbidos (tinidos), além de instabilidade emocional com ansiedade. Pacientes com mal de Alzheimer podem apresentar uma modesta melhora no desempenho cognitivo e social (Clayton \& Stock, 2006, Thomas \& Thomas, 2004).

De acordo com Fernandes (2005), tanto no Brasil, quanto em outros países o Ginkgo foi estudado como opção terapêutica nas demências do tipo Alzheimer e multi-infarto. Os resultados de uma meta-analise de quatro ensaios clínicos mostraram que o extrato padronizado de Ginkgo EGb 761 produziu um efeito positivo, significativo sobre a função cognitiva em pacientes com doença Alzheimer. Esses resultados foram comparáveis aos do donepezil, um inibidor da colinesterase utilizando no tratamento desta doença. Soma-se a isto, outro estudo envolvendo 50 pacientes com doença de Alzheimer, demonstrou uma melhora da sociabilidade, da depressão, da distração e do esquecimento (Lima \& David, 2006).

\section{Metodologia}

O estudo foi executado através de uma revisão sistemática, considerando a relevância do tema, buscando conhecer sob o olhar de alguns autores bem como analisar como e quais as interações medicamentosas do fitoterápico G. biloba. Segundo Foglito (2007) a revisão sistemática, é aquela que reúne ideias oriundas de diferentes fontes, visando construir uma nova teoria ou uma nova forma de apresentação para um assunto já conhecido.

A elaboração da pesquisa teve por ferramenta embasadora material já publicado sobre o tema; artigos científicos em 
publicações periódicas e disponíveis nos seguintes bancos de dados: Scielo e Pubmed, sendo considerados os artigos publicados entre 2010-2020, que relatavam sobre as possíveis interações medicamentosas do fitoterápico G. biloba.

As palavras-chave utilizadas para pesquisa foram: interações medicamentosas, G. biloba e fitoterápicos. Foram adotados os seguintes critérios de inclusão: artigos escritos em Português, com disponibilidade de texto completo em suporte eletrônico publicado em periódicos nacionais e os critérios de exclusão: teses, capítulos de tese, livros, capítulos de livros, anais de congressos ou conferencias, relatórios técnicos e científicos e documentos ministeriais.

Foi construído um banco de dados alimentado por meio das análises obtidas da pesquisa, no qual foram organizados em programa Microsoft Word 2019. Após a identificação dos artigos, nas fontes de busca mencionadas, foram avaliados os títulos e resumos, de modo a seleciona-los. Foram elencados os artigos que faziam parte da amostra e estes foram registrados em ficha própria contendo dados do periódico, base de dados, ano de publicação, objetivos, resultados e conclusões.

\section{Resultados e Discussão}

Da pesquisa bibliográfica efetuada obteve um total 497 artigos publicados entre os anos de 2010 a 2020. Após leitura, selecionou-se sete trabalhos, iniciando leituras exaustivas dos textos, fazendo uma síntese de cada artigo e categorizando os tipos de interações medicamentosas relacionadas a G. biloba. A distribuição do número de publicações pelo ano de publicação está disposta na Tabela 1.

Tabela 1. Distribuição do número de publicações pelo ano de publicação.

\begin{tabular}{ccc}
\hline Periódico & Autor & Tipo de estudo \\
\hline Colloquium Vitae & Silva et al., 2010 & Pesquisa experimental \\
\hline Revista Saúde e Desenvolvimento & Carneiro e Comarella (2016) & Revisão bibliográfica \\
\hline Revista de Pesquisa: Cuidado é Fundamental Online & Gelatti et al. (2016) & Pesquisa experimental \\
\hline Revista Saúde & Nicolleti et al. (2010) & Revisão bibliográfica \\
\hline
\end{tabular}

Fonte: Autores.

Foi possível identificar que os anos com maiores concentrações de publicações em relação as interações medicamentosas foi o de 2016 com dois trabalhos. Referente ao tipo de estudo com maior concentração de publicações, o predomínio foi de pesquisa bibliográfica, seguido por estudo experimental. Silva et al. (2010) constatou que O Ginkgo pode potencializar a ação de agentes antitrombóticos (antiplaquetários e anticoagulantes, incluindo a heparina e varfarina) e aumentar o risco de hemorragia intracerebral. A administração de Ginkgo e ticlodipina aumenta o tempo de hemorragia em 150\% (P.R. VADEMÉCUM DE MEDICAMENTOS, 2004).

Carneiro e Comarella (2016) ressaltam a interação entre o ginkgo com anticoagulante ou antiplaquetário oral, como a varfarina, podendo aumentar o risco de hemorragia, já que o Ginkgolídeo B pode inibir o fator de ativação plaquetária inibindo a sua agregação. Os autores relatam ainda que o uso de ginkgo concomitante com o anti-hipertensivo nifedipina aumenta as reações adversas deste, como dor de cabeça, rubor e edema de tornozelo. Alexandre e colaboradores (2008) realizaram um estudo sobre as interações entre fármacos e medicamentos fitoterápicos elaborados com ginkgo (G. biloba) e ginseng (Panax ginseng C. A. Mey. e Panax quinquefolius L.). A partir disso, destaca-se a interação entre o ginkgo com anticoagulante ou antiplaquetário oral, como a varfarina, podendo aumentar o risco de hemorragia, já que o Ginkgolídeo B pode inibir o fator de ativação plaquetária inibindo a sua agregação. Os autores relatam ainda que o uso de ginkgo concomitante com o antihipertensivo nifedipina aumenta as reações adversas deste, como dor de cabeça, rubor e edema de tornozelo.

Gellati e Oliveira (2016) na busca em Identificar as potenciais interações decorrentes do uso de plantas medicinais e fitoterápicos concomitante a medicamentos utilizados por mulheres climatéricas, ressaltaram que nesse estudo o G. biloba foi o 
fitoterápico mais envolvido em interações, provavelmente porque foi o mais utilizado pelas mulheres devido a sua ampla divulgação pela mídia, e pelo fato de a planta afetar várias isoenzimas do citocromo P450.

Nicoleti et al. (2010) relata prováveis interações medicamentosas decorrentes do uso das principais drogas de origem vegetal como recurso aditivo de informações para facilitar a orientação correta quanto ao uso racional de medicamentos. Dessa forma, o uso de ginkgo tem possibilidade de potencializar a ação do ácido acetilsalicílico e do clopidogrel, de anticoagulantes como varfarina e heparina, além de anti-inflamatórios não esteroidais como ibuprofeno ou naproxeno, aumentando, assim, o risco de sangramentos. Outro fato que pode justificar a maior frequência de interações relacionadas com esse fitoterápico refere-se ao seu uso milenar e às inúmeras pesquisas relacionadas com este produto (Gellati \& oliveira, 2016).

Assim, as interações que ocorrem entre fármacos e os componentes químicos presentes nas plantas medicinais e nos medicamentos fitoterápicos podem acarretar em alterações nas concentrações plasmáticas dos fármacos e, consequentemente, mudanças nos seus perfis de eficácia e/ou segurança (Alexandre, et al., 2007). Vários casos são mencionados sobre prováveis interações com fármacos anticoagulantes orais, antiplaquetários, anti-inflamatórios não-esteroidais, anticonvulsivantes, antidepressivos, anti-hipertensivos e antiulcerosos (Alexandre, et al., 2007).

A literatura é rica em vários estudos sobre as interações envolvendo o ginkgo, todavia, não são conclusivos, além de que alguns deles demonstram resultados contraditórios (Nicoleti, et al., 2007). Baseado em dados de laboratório e pesquisa em humanos, o uso de ginkgo poderá diminuir a pressão sanguínea embora haja relato de elevação de pressão em indivíduo que estava tomando diurético à base de tiazida (Nicoleti, et al., 2007).

A frequência no uso de plantas medicinais na atualidade vem de uma cultura popular, trazida através dos tempos, que acaba levando a um uso irracional dessas plantas medicinais, já que a população tem fácil acesso a essas e ainda têm a percepção que não trazem efeitos indesejáveis (Nicoletti, et al., 2007).

Em relação as plantas medicinais, a influência direta da medicina ocidental moderna oportunizada pelos centros e profissionais da saúde somada à facilidade de acesso aos medicamentos alopáticos por meios das farmácias presentes nas áreas urbanas, são alguns dos fatores que afetam o conhecimento e práticas da medicina local (Santos, et al., 2016).

Apesar disso, os recursos vegetais para fins terapêuticos em áreas urbanas ainda têm sido fortemente empregados (Santos et al., 2016). Nestas áreas, eles são geralmente encontrados nos quintais e mercados públicos, espaços urbanos que oferecem alternativas terapêuticas mais baratas quando comparadas ao alto custo dos medicamentos alopáticos (Rao, et al., 2004).

Atualmente, a utilização de plantas medicinais e fitoterápicos é uma prática mundialmente disseminada, sendo encorajada pela Organização Mundial de Saúde (OMS), especialmente em países em desenvolvimento. No Brasil, o uso de plantas medicinais no tratamento de enfermidades tem influências da cultura indígena, africana e europeia, cujas marcas foram integradas em um conjunto de princípios que visam à cura de doenças e restituem ao homem a vida natural (Silva, et al., 2008). Mesmo com o incentivo da indústria farmacêutica para a utilização de medicamentos industrializados, grande parte da população ainda faz uso de práticas terapêuticas no cuidado à saúde, como as plantas medicinais, utilizadas para aliviar ou mesmo curar algumas enfermidades (Badke, et al., 2012).

\section{Considerações Finais}

A utilização de plantas medicinais e medicamentos fitoterápicos tem aumentado nos últimos anos, principalmente, pelos portadores de doenças crônicas. Como as plantas medicinais e os medicamentos fitoterápicos são caracterizados por uma mistura complexa de componentes químicos e podem apresentar diversos mecanismos de ação, não há dúvidas de que, quando administrados concomitantemente, podem interagir com diversos fármacos, alterando os seus perfis de eficácia e segurança. No Brasil, tem aumentado mais a cada dia o consumo de fitoterápicos, devido a divulgação de dados imprecisos de trabalhos 
científicos, os quais demonstram algum efeito preventivo ou terapêutico dessas plantas medicinais. Essas informações, em conjunto com a medicina popular, geram uma tendência na população de interpretar que formulações medicamentosas com plantas medicinais não são prejudiciais à saúde e nem tóxicas, só por serem provenientes de plantas. Assim, a pesquisa serve como subsídio para futuros estudos acerca de fitoterápicos.

\section{Referências}

Alexandre, R. F., Bagatini, F., \& Simões, C. M. O. (2008). Interações entre fármacos e medicamentos fitoterápicos à base de ginkgo ou ginseng. Revista Brasileira de Farmacognosia, 18.117-127.

Balunas, M. J., \& Kinghorn, A. D. (2005). Drug discovery from medicinal plants. Life Sciences, 78, 431-441.

Barreto, A. G., Biscaia, E. C., Veiga, V. F., Pinto, A. C., Carvalhaes, S. F., \& Maciel, M. A. M. (2005). Cromatografia de troca-iônica aplicada ao isolamento da fração ácida do óleo de copaíba (Copaifera multijuga) e da sacaca (Croton cajucara). Química Nova, 28(4), 719-722.

Belwal, T., Giri, L., Bahukhandi, A., Tariq, M., Kewlani, P., Bhatt, I. D., \& Rawal, R. S. (2019). Ginkgo biloba. In S. M. Nabavi \& A. S. Silva (Eds.), Nonvitamin and nonmineral nutritional supplement, 241-250.

Carneiro, A. L., \& Comarella, L. (2016). Principais Interações Entre Plantas. Revista Saúde e desenvolvimento, 9(5).

Carvalho, J. I. \& Rocha, M. S. Interações Medicamentosas dos Fitoterápicos Ginkgo biloba, Panax ginseng e Hypericum perforatum com medicamentos alopáticos. Centro de Pós-Graduação Oswaldo Cruz.

Crane, P. R., Nagata, T., Murata, J., Ohi-Toma, T., Duval, A., Nesbitt, M., \& Jarvis, C. (2013). Ginkgo bilobae Connections with people and art across a thousand years. Botanical Magazine, 30, 239e260.

De Pasquale, A. (1984). Pharmacognosy: oldest modern science. Journal of Ethnopharmacology, 11. 1-6.

Dias, E. C. M., Trevisan, D. D., Cappelleti, S. N., Amorim, N. R., \& Silva, E. M. (2017). Uso de fitoterápicos e potenciais riscos de interações medicamentosas: reflexões para prática segura. Revista Baiana de Saúde Pública, 41. 1-10.

Fogliatto, F. (2007). Users/ANA/Downloads/MargheritaMaschietto GuerraTCC\%20(1).

França, I. S. X., Souza, J. A., Baptista, R. S., \& Britto, V. R.S. (2008). Medicina popular: benefícios e malefícios das plantas medicinais. Revista Brasileira de Enfermagem, 61(2), 201-208.

Gelatti, G. T., Oliveira, K. R. O., \& Colet, C. F. (Potenciais interações relacionadas ao uso de medicamentos, plantas medicinais e fitoterápicos em mulheres no período do climatério. J. res.: fundam. Care, 8 (2), 4328-4346.

Hassan, A. R. B. (2012). Medicinal plants (importanceand uses). Pharmaceut Anal Acta, 3, 10-20.

Kinghorn, A. D., Farnsworth, N. R., Soejarto, D. D., Cordell, G. A., Swanson, S. M., Pezzuto, J. M., Wani, M. C., Wall, M. E., Oberlies, N. H., Kroll, D.J., Kramer, R. A., Rose, W. C., Vite, G. D., Fairchild, C. R., Peterson, R. W. \& Wild, R. (2003). Novel strategies for the discovery of plant-derived anticancer agents. Pharmaceutical Biology. 41. 53-67.

Lombardo, M. (2018). Potencial Adverso de Medicamentos Fitoterápicos: Um Estudo com Foco em Medicamentos de Registro Simplificado. Rev Ciên Saúde, $3(1) .1$.

Nascimento, B. J., Tínel, L. O., Silva, E. S., Rodrigues, L. A., Freitas, T. O. N., Nunes, X. P., \& Amorim, E. L. C. (2016). Avaliação do conhecimento e percepção dos profissionais da estratégia de saúde da família sobre o uso de plantas medicinais e fitoterapia em Petrolina-PE, Brasil.. Revista Brasileira de Plantas Medicinais, 18(1), 57-66.

Navarro, D. (2005). Estudo Químico, Biológico e Farmacológico das espécies Allamanda blanchetti e Allamandaschottii na obtenção de moléculas bioativas de potencial terapêutico.

Nicoletti, M. A., Oliveira, M. A., Bertasso, C. C., Caporossi, P. Y., \& Tavares, A. P. L. (2007). Principais interações no uso de medicamentos fitoterápicos. Revista Infarma, 19, 32-40.

Nicoletti, M. A., Carvalho, K. C., Oliveira, J. R. M. A., Bertasso, C. C., Caporossi, P. Y., \& Tavares, A. P. L. (2010). Uso popular de medicamentos contendo drogas de origem vegetal e/ou plantas medicinais: principais interações decorrentes. Revista Saúde, 4(1).

Sanchita, A., Sharma, A. (2018). Gene expression analysis in medicinal plants underabiotic stress conditions. Plant Metabolites and Regulation Under Environmental, 30. 407-414.

Silva, T. F. O., Marcelino, C. E., \& Gomes. A. J. P. S. (2010). Utilizações e Interações Medicamentosas de Produtos Contendo o Ginkgo biloba. Colloquium Vitae, 2 (1), 54-61.

Silva, F. L. A., Oliveira, R. A. G., \& Araújo, E. C. (2008). Use of medicinal plants by the elders at a family health estrategy. Rev Enferm UFPE on line. 2(1):916.

Veiga, V. F., Pinto, A. C., \& Maciel, M. A. M. (2005). Plantas medicinais: cura segura. Química nova. 28(3), 519-528. 
Research, Society and Development, v. 10, n. 6, e13810615535, 2021

(CC BY 4.0) | ISSN 2525-3409 | DOI: http://dx.doi.org/10.33448/rsd-v10i6.15535

Zhang, Z., MEI, N., Chen, S., Guo, L., \& Guo, X. (2016). Assessment of Genotoxic Effects of Selected Herbal Dietary Supplements. Nutraceuticals. Elsevier $883 \mathrm{e} 892$.

Cuassolo, F., Ladio, A., \& Ezcurra, C. (2010). Aspectos de la comercialización y control de calidad de las plantas medicinales más vendidas en una comunidad urbana del NO de la Patagonia Argentina. Boletín Latinoamericano y del Caribe de Plantas Medicinales y Aromáticas, 9(3),165-176.

Hilgert, N. I., Higuera, M. L., \& Kristensen, M. J. (2010). La medicina herbolaria en el contexto urbano. Estudio de caso en un barrio de la ciudad de Tandil, Argentina. Boletín Latinoamericano y del Caribe de Plantas Medicinales y Aromáticas, 9(3),177-190.

Pirondo, A., Coulleri, J. P., Keller, H. A., \& Ferrucci, M. S. (2011). Influencia de factores externos sobre la comercialización de plantas medicinales en un medio urbano: el caso de vendedores criollos e indígenas en Corrientes, Argentina. Boletin Latinoamericano y del Caribe de Plantas Medicinales y Aromaticas, 10(6), 553-569.

Ladio, A. H., Molares, S., Ochoa, J. J. I., \& Cardoso, B. (2013). Etnobotánica aplicada en Patagonia: la comercialización de malezas de uso comestible y medicinal en una feria urbana de San Carlos de Bariloche (Río Negro, Argentina). Boletin Latinoamericano y del Caribe de Plantas Medicinales y Aromaticas, 12 (1), 24-37.

Santos, A. B. N., Araujo, M. P., Sousa, R.S., \& Lemos, J. R. (2016). Plantas medicinais conhecidas na zona urbana de Cajueiro da Praia, Piauí, Nordeste do Brasil. Rev. bras. plantas med. 18(2), 442-450.

Parente, C. E. T., \& Rosa, M. M. T. (2001). Plantas comercializadas como medicinais no município de Barra do Piraí, RJ. Rodriguésia, 52, 47-59.

Rao, M., Palada, M. \& Becker, B. (2004). Medicinal and aromatic plants in agroflorestry systems. Agroflorestry Systems, 1(61), 107-122.

Badke, M. R., Budó, M. L. D., Alvim, N. A. T., Zanetti, G. D., \& Heisler, E. V. (2012). Saberes e práticas populares de cuidado em saúde com o uso de plantas medicinais .Texto \& Contexto Enferm, 21(2). 363-70. 